In A. Sarychev A. Shiryaev, M. Guerra and M. R. Grossinho (eds): Mathematical Control Theory and Finance, Springer, (2008), pp. 71-94,

On option-valuation in illiquid markets: invariant solutions to a nonlinear model

L. A. BORDAG

HALMSTAD UNIVERSiTy, BOX 823, 301

Authors version 


\title{
On option-valuation in illiquid markets: invariant solutions to a nonlinear model
}

\author{
L. A. BORDAG \\ Halmstad University, Box 823, 30118 Halmstad, Sweden \\ EMAIL: Ljudmila.Bordag@hh.se \\ URL: http://www2.hh.se/staff/ljbo/
}

\begin{abstract}
The present model describes a perfect hedging strategy for a large trader. In this case the hedging strategy affects the price of the underlying security. The feedback-effect leads to a nonlinear version of the Black-Scholes partial differential equation. Using Lie group theory we reduce in special cases the partial differential equation to some ordinary differential equations. The Lie group found for the model equation gives rise to invariant solutions. Families of exact invariant solutions for special values of parameters are described.
\end{abstract}

1. Introduction. One of the basic assumptions of the Black-Scholes option theory is that all participants act on the market as price takers. Recently a series of papers [Frey, 1998] , [Frey, 2000], [Schonbucher and Wilmott, 2000], [Sircar and Papanicolaou, 1998] appeared in which this assumption has been relaxed. In these models the hedging strategy affects the price of the underlying security. For a large trader a hedge-cost of the claim differs from the price of the option for price takers.

In [Frey, 1998], [Frey, 2000] Frey developed a model of market illiquidity. He describes the asset price dynamics which results if a large trader chooses a given stock-trading strategy $\left(\alpha_{t}\right)_{t}$. The admissible class of stock trading strategies includes the left-continuous stock-holdings $\left(\alpha_{t}\right)_{t}$ for which the rightcontinuous process with $\alpha_{t}^{+}=\lim _{s \rightarrow t, s>t} \alpha_{s}$ is a semimartingale. If the large trader uses a particular trading strategy $\alpha^{+}$then, as expected, $\mathrm{d} \alpha_{t}^{+}>0$ leads to $\mathrm{d} S_{t}>0$ and correspondingly $\mathrm{d} \alpha_{t}^{+}<0$ leads to $\mathrm{d} S_{t}<0$, where $S_{t}$ denotes the asset price process. Frey's model has the form of the stochastic differential equation

$$
\mathrm{d} S_{t}=\sigma S_{t-} \mathrm{d} W_{t}+\rho S_{t-} \mathrm{d} \alpha_{t}^{+},
$$

where $\sigma>0$ is a constant volatility, $W_{t}$ is a standard Brownian motion, $S_{t-}$ denotes the left limit $\lim _{s \rightarrow t, s<t} S_{t}$, and $\rho$ is the market illiquidity parameter with $0<\rho<1$. If we denote the filtration associated to the Brownian motion by $\mathcal{F}(t)$ than a hedge cost of the claim $u(S, t)$ is given by a conditional expectation $u(S, t)=\mathrm{E}[h(S) \mid \mathcal{F}(t)]$ for any Borel-measurable function $h(S)$. The function $h(S)$ is the payoff at time $T$ of a derivative security $u(S, t)$ whose underlying asset price process is (1). After the Feynman-Kac theorem [Shreve, 2004] together with a fixed point argument [Frey, 2000] $u(S, t)$ satisfies the corresponding partial differential equation

$$
u_{t}+\frac{\sigma^{2} S^{2}}{2} \frac{u_{S S}}{\left(1-\rho S u_{S S}\right)^{2}}=0,
$$

and the terminal condition $u(S, T)=h(S)$ for all $S$ where $S \geq 0, t \in[0, T], T>0$.

The value $1 /\left(\rho S_{t}\right)$ is called the depth of the market at time $t$. If $\rho \rightarrow 0$ then simultaneously $\mathrm{d} \alpha_{t}^{+} \rightarrow 0$ and the equation (2) reduces to the Black-Scholes case.

In model (1) the parameter $\rho$ is a characteristics of the market and does not depend on the payoff of hedged derivatives. The value of $\rho$ is fixed during the trading process and can be estimated in different ways, in the papers [Frey, 1998], [Frey, 2000] the value $\rho$ is equal to 0.1,0.2,0.3,0.4.

In the paper by Frey and Stremme, [Frey and Stremme, 1997], a heterogeneous portfolio was introduced, and a model with variable volatility was studied.

Later on, in [Frey and Patie, 2002] Frey and Patie followed another approach and examined the feedback effect of the option replication strategy of the large trader on the asset price process. They obtain a new model by introduction of a liquidity coefficient which depends on the current stock price. 
It means the stochastic equation now takes the form

$$
\mathrm{d} S_{t}=\sigma S_{t-} \mathrm{d} W_{t}+\rho \lambda\left(S_{t-}\right) S_{t-} \mathrm{d} \alpha_{t}^{+},
$$

where $\lambda: R^{+} \rightarrow R^{+}$is a continuous bounded function for all $S \geq 0$. In this model the depth of the market at time $t$ is defined as $1 /\left(\rho \lambda\left(S_{t}\right) S_{t}\right) ; \lambda(S)$ is called level-depended liquidity profile.

The Feynman-Kac theorem together with a fixed point argument [Frey and Patie, 2002] leads in case of (3) to a partial differential equation which is similar to the equation (2) where the constant $\rho$ is replaced by $\rho \lambda(S)$. The values of $\rho$ and $\lambda(S)$ may be estimated from the observed option prices and depend on the payoff $h(S)$.

The feedback-effect described in (3) leads to a nonlinear version of the Black-Scholes partial differential equation,

$$
u_{t}+\frac{\sigma^{2} S^{2}}{2} \frac{u_{S S}}{\left(1-\rho S \lambda(S) u_{S S}\right)^{2}}=0,
$$

with $S \in[0, \infty), \quad t \in[0, T]$. As usual, $S$ denotes the price of the underlying asset and $u(S, t)$ denotes the hedge-cost of the claim with a payoff $h(S)$ which will be defined later. The hedge-cost is different from the price of the derivatives product in illiquid markets. In the sequel $t$ is the time variable, $\sigma$ defines the volatility of the underlying asset.

In [Frey and Patie, 2002] some examples for estimates of $\rho$ and $\lambda(S)$ were matched for the call spread and for the European call. The values $\rho$ are equal to 0.01, 0.02, 0.05, 0.1, 0.2, 0.4 and the level-depended liquidity profile $\lambda(S)$ is approximated by a quadratic function of $S$. We shall concentrate our investigations on the non-trivial case $\rho \neq 0$.

Depending on the assumptions concerning the market, different variations of the Black-Scholes formula can arise like in the well known models [Schonbucher and Wilmott, 2000],

[Sircar and Papanicolaou, 1998]. Usually the volatility term in the Black-Scholes formula will be replaced in order to fit the behavior of the price on the market. The modeling process is not concluded now and new models can appear. For those of the type (2), (4), or in [Sircar and Papanicolaou, 1998] the appearance of the small parameter multiplying the highest derivative is crucial. The nonlinear parabolic equations with a singular perturbation describe usually a richer class of phenomena than equations with a regular perturbation. An analytical study of these equations may be useful for an easier classification of models.

Frey and co-authors studied equation (4) under some restrictions and did some numerical simulations. Our goal is to investigate this equation using analytical methods.

We study the model equation (4) by means of Lie group theory. We used the method of Lie group analysis to investigate other financial models given by nonlinear partial differential equations in [Bordag and Chmakova, 2007], [Bordag, 2005], [Chmakova, 2005] [Bordag, 2006], [Bordag and Frey, 2009]. This method has a long tradition beginning with the work of S. Lie [Lie, 1912]. Its applications are based on local symmetries. A modern description of the method as well as a large number of applications can be found in [Ovsiannikov, 1982], [Olver, 1986], [Stephani, 1994], [Gaeta, 1994], [Ibragimov, 1999].

In Section 2 we find the Lie algebra and global equations for the symmetry group of equation (4). For a special form of the function $\lambda(S)$ it is possible to find two functionally independent invariants of the symmetry group.

Using the symmetry group and its invariants we reduce the partial differential equation (4) in some special cases to ordinary differential equations in Section 3. We shell study singular points of the reduced equations in Section 4 and describe the behavior of the invariant solutions. For a fixed set of parameters the complete set of exact invariant solutions is given. It is important to obtain exact solutions for the model (4) to use invariant solutions for instance as a test case for various numerical methods.

Further, in Section 5 we study different properties of the invariant solutions and their sensitivity with respect to the illiquidity parameter. In particular, if any terminal conditions are fixed, $u(S, T)=h(S)$, 
then the value $u(S, t)$ will increase if the value of the parameter $\rho$ increases, i.e. hedge costs of the large trader on the market depends in expected way on the position of the large trader. We obtain a typical terminal payoff function for these solutions if we just fix $t=T$. By changing the value of the integrating constants and by adding a linear function of $S$ we are able to modify terminal payoff functions for the solutions. Hence we can approximate typical payoff profiles of some financial derivatives quite well.

2. Lie group symmetries. Let us introduce a two-dimensional space $X$ of independent variables $(S, t) \in X$ and a one-dimensional space of dependent variables $u \in U$. We consider the space $U_{(1)}$ of the first derivatives of the variable $u$ on $S$ and $t$, i.e., $\left(u_{S}, u_{t}\right) \in U_{(1)}$ and analogously we introduce the space $U_{(2)}$ of the second order derivatives $\left(u_{S S}, u_{S t}, u_{t t}\right) \in U_{(2)}$. We denote by $M=X \times U$ a base space which is a Cartesian product of pairs $(x, u)$ with $x=(S, t) \in X, u \in U$. The differential equation (4) is of the second order and to represent this equation as an algebraic equation we introduce a second order jet bundle $M^{(2)}$ of the base space $M$, i.e.,

$$
M^{(2)}=X \times U \times U_{(1)} \times U_{(2)}
$$

with a natural contact structure. We label the coordinates in the jet bundle $M^{(2)}$ by $w=\left(S, t, u, u_{S}, u_{t}, u_{S S}, u_{S t}, u_{t t}\right) \in M^{(2)}$.

In the space $M^{(2)}$ equation (4) is equivalent to the relation

$$
\Delta(w)=0, \quad w \in M^{(2)},
$$

where we denote by $\Delta$ the following function

$$
\Delta\left(S, t, u, u_{S}, u_{t}, u_{S S}, u_{S t}, u_{t t}\right)=u_{t}+\frac{\sigma^{2} S^{2}}{2} \frac{u_{S S}}{\left(1-\rho S \lambda(S) u_{S S}\right)^{2}} .
$$

We identify the algebraic equation (6) with its solution manifold $L_{\Delta}$ defined by

$$
L_{\Delta}=\left\{w \in M^{(2)} \mid \Delta(w)=0\right\} \subset M^{(2)} .
$$

Let us consider an action of a Lie-point group on our differential equation and its solutions. We define a symmetry group $G_{\Delta}$ of equation (6) by

$$
G_{\Delta}=\left\{g \in \operatorname{Diff}\left(\mathrm{M}^{(2)}\right) \mid \mathrm{g}: \mathrm{L}_{\Delta} \rightarrow \mathrm{L}_{\Delta}\right\}
$$

consequently we are interested in a subgroup of $\operatorname{Diff}\left(\mathrm{M}^{(2)}\right)$ which is compatible with the structure of $L_{\Delta}$.

As usual we first find the corresponding symmetry Lie algebra $\operatorname{Diff}_{\Delta}\left(M^{(2)}\right) \subset \mathcal{D}$ iff $\left(M^{(2)}\right)$ and then use the main Lie theorem to obtain $G_{\Delta}$ and its invariants.

We denote an element of a Lie-point vector field on $M$ by

$$
V=\xi(S, t, u) \frac{\partial}{\partial S}+\tau(S, t, u) \frac{\partial}{\partial t}+\phi(S, t, u) \frac{\partial}{\partial u},
$$

where $\xi(S, t, u), \tau(S, t, u)$ and $\phi(S, t, u)$ are smooth functions of their arguments, $V \in \operatorname{Diff}(M)$. The operators (10) are called as well infinitesimal generators.

If the infinitesimal generators of $g \in G_{\Delta}(M)$ exist then they have the structure of type (10) and form an Lie algebra $\mathcal{D}$ if $f_{\Delta}(M)$.

A Lie group of transformations $G_{\Delta}(M)$ acting on the base space $M$ induce as well the transformations on $M^{(2)}$ which we denoted by $G_{\Delta}$.

The corresponding Lie algebra $\mathcal{D}$ if $f_{\Delta}\left(M^{(2)}\right)$ will be composed of vector fields

$$
\begin{aligned}
p r^{(2)} V & =\xi(S, t, u) \frac{\partial}{\partial S}+\tau(S, t, u) \frac{\partial}{\partial t}+\phi(S, t, u) \frac{\partial}{\partial u} \\
& +\phi^{S}(S, t, u) \frac{\partial}{\partial u_{S}}+\phi^{t}(S, t, u) \frac{\partial}{\partial u_{t}} \\
& +\phi^{S S}(S, t, u) \frac{\partial}{\partial u_{S S}}+\phi^{S t}(S, t, u) \frac{\partial}{\partial u_{S t}}+\phi^{t t}(S, t, u) \frac{\partial}{\partial u_{t t}},
\end{aligned}
$$


which are the second prolongation of vector fields $V$. Here the smooth functions $\phi^{S}(S, t, u), \phi^{t}(S, t, u), \phi^{S S}(S, t, u), \phi^{S t}(S, t, u)$ and $\phi^{t t}(S, t, u)$ are uniquely defined by the functions $\xi(S, t, u), \tau(S, t, u)$ and $\phi(S, t, u)$ using the prolongation procedure (see [Ovsiannikov, 1982], [Olver, 1986], [Stephani, 1994], [Gaeta, 1994], [Ibragimov, 1999]).

Theorem 2.1. The differential equation (4) with an arbitrary function $\lambda(S)$ possesses a trivial three dimensional Lie algebra Dif $f_{\Delta}(M)$ spanned by infinitesimal generators

$$
V_{1}=\frac{\partial}{\partial t}, \quad V_{2}=S \frac{\partial}{\partial u}, \quad V_{3}=\frac{\partial}{\partial u} .
$$

Only for the special form of the function $\lambda(S) \equiv \omega S^{k}$, where $\omega, k \in R$ equation (4) admits a non-trivial four dimensional Lie algebra spanned by generators

$$
V_{1}=\frac{\partial}{\partial t}, \quad V_{2}=S \frac{\partial}{\partial u}, \quad V_{3}=\frac{\partial}{\partial u}, V_{4}=S \frac{\partial}{\partial S}+(1-k) u \frac{\partial}{\partial u} .
$$

Proof. The symmetry algebra $\mathcal{D}$ if $f_{\Delta}\left(M^{(2)}\right)$ of the second order differential equation $(6)$ can be found as a solution to the determining equations

$$
p r^{(2)} V(\Delta)=0(\bmod (\Delta=0))
$$

i.e., the equation (12) should be satisfied on the solution manifold $L_{\Delta}$.

For our calculations we will use the exact form of the coefficients $\phi^{t}(S, t, u)$ and $\phi^{S S}(S, t, u)$ only. The coefficient $\phi^{t}(S, t, u)$ can be defined by the formula

$$
\phi^{t}(S, t, u)=\phi_{t}+u_{t} \phi_{u}-u_{S} \xi_{t}-u_{S} u_{t} \xi_{u}-u_{t} \tau_{t}-\left(u_{t}\right)^{2} \tau_{u},
$$

and the coefficient $\phi^{S S}(S, t, u)$ by the expression

$$
\begin{aligned}
\phi^{S S}(S, t, u) & =\phi_{S S}+2 u_{S} \phi_{S u}+u_{S S} \phi_{u} \\
& +\left(u_{S}\right)^{2} \phi_{u u}-2 u_{S S} \xi_{S}-u_{S} \xi_{S S}-2\left(u_{S}\right)^{2} \xi_{S u} \\
& -3 u_{S} u_{S S} \xi_{u}-\left(u_{S}\right)^{3} \xi_{u u}-2 u_{S t} \tau_{S}-u_{t} \tau_{S S} \\
& -2 u_{S} u_{t} \tau_{S u}-\left(u_{t} u_{S S}+2 u_{S} u_{S t}\right) \tau_{u}-\left(u_{S}\right)^{2} u_{t} \tau_{u u}
\end{aligned}
$$

where the subscripts by $\xi, \tau, \phi$ denote corresponding partial derivatives.

The first equations of the set (12) imply that if $V \in \mathcal{D}$ if $f_{\Delta}(M)$ then

$$
\xi(S, t, u)=a_{1} S, \quad \tau(S, t, u)=a_{2}, \quad \phi(S, t, u)=a_{3} S+a_{4}+a_{5} u,
$$

where $a_{1}, a_{2}, a_{3}, a_{4}, a_{5}$ are arbitrary constants and $\xi, \tau, \phi$ are coefficients in the expression (10).

The remaining equation has a form

$$
a_{1} S \lambda_{S}(S)-\left(a_{1}-a_{5}\right) \lambda(S)=0
$$

Because this equation should be satisfied for all $S$ identically we obtain for an arbitrary function $\lambda(S)$

$$
a_{1}=a_{5}=0, \quad \rightarrow \xi(S, t, u)=0, \quad \tau(S, t, u)=a_{2}, \quad \phi(S, t, u)=a_{3} S+a_{4} .
$$

Finally, $\mathcal{D}$ if $f_{\Delta}(M)$ admits the following infinitesimal generators

$$
V_{1}=\frac{\partial}{\partial t}, \quad V_{2}=S \frac{\partial}{\partial u}, \quad V_{3}=\frac{\partial}{\partial u},
$$

with commutator relations

$$
\left[V_{1}, V_{2}\right]=\left[V_{1}, V_{3}\right]=\left[V_{2}, V_{3}\right]=0 \text {. }
$$


If the function $\lambda(S)$ has a special form

$$
\lambda(S) \equiv \omega S^{k}, \quad \omega, k \in R
$$

then the equation (16) on the coefficients of (10) is less restrictive and we obtain

$$
\xi(S, t, u)=a_{1} S, \quad \tau(S, t, u)=a_{2}, \quad \phi(S, t, u)=(1-k) a_{1} u+a_{3} S+a_{4} .
$$

Now the symmetry algebra $\mathcal{D} i f f_{\Delta}(M)$ admits four generators

$$
V_{1}=\frac{\partial}{\partial t}, \quad V_{2}=S \frac{\partial}{\partial u}, \quad V_{3}=\frac{\partial}{\partial u}, V_{4}=S \frac{\partial}{\partial S}+(1-k) u \frac{\partial}{\partial u},
$$

with commutator relations

$$
\begin{array}{r}
{\left[V_{1}, V_{2}\right]=\left[V_{1}, V_{3}\right]=\left[V_{1}, V_{4}\right]=\left[V_{2}, V_{3}\right]=0,} \\
{\left[V_{2}, V_{4}\right]=-k V_{2}, \quad\left[V_{3}, V_{4}\right]=(1-k) V_{3} .}
\end{array}
$$

Remark 2.2. In the general case the algebra (22) possesses a three dimensional Abelian sub-algebra and one dimensional center. For the cases $k=0,1$ the center is two dimensional (23) and we see later that the corresponding equations (44) became autonomous.

The symmetry algebra $\mathcal{D}$ if $f_{\Delta}(M)$ defines by the Lie equations [Lie, 1912] the corresponding symmetry group $G_{\Delta}$ of the equation (6). To find the global form of transformations for the solutions to equation (4) corresponding to this symmetry group we just integrate the system of ordinary differential equations

$$
\begin{aligned}
& \frac{d \tilde{S}}{d \epsilon}=\xi(\tilde{S}, \tilde{t}, u), \\
& \frac{d \tilde{t}}{d \epsilon}=\tau(\tilde{S}, \tilde{t}, \tilde{u}), \\
& \frac{d \tilde{u}}{d \epsilon}=\phi(\tilde{S}, \tilde{t}, \tilde{u}),
\end{aligned}
$$

with initial conditions

$$
\left.\tilde{S}\right|_{\epsilon=0}=S,\left.\tilde{t}\right|_{\epsilon=0}=t,\left.\tilde{u}\right|_{\epsilon=0}=u \text {. }
$$

Here the variables $\tilde{S}, \tilde{t}$ and $\tilde{u}$ denote values $S, t, u$ after a symmetry transformation. The parameter $\epsilon$ describes a motion along an orbit of the group.

Theorem 2.3. The action of the symmetry group $G_{\Delta}(M)$ of (4) with an arbitrary function $\lambda(S)$ is given by (28)-(30). If the function $\lambda(S)$ has the special form (20) then the action of the symmetry group $G_{\Delta}(M)$ is represented by (31)-(33).

Proof. The solutions to the system of ordinary differential equations (24) with functions $\xi, \tau, \phi$ defined by (17) and initial conditions (27) have the form

$$
\begin{aligned}
\tilde{S} & =S, \\
\tilde{t} & =t+a_{2} \epsilon, \\
\tilde{u} & =u+a_{3} S \epsilon+a_{4} \epsilon, \quad \epsilon \in(-\infty, \infty) .
\end{aligned}
$$

The equations (28)-(30) are the global representation of the symmetry group $G_{\Delta}$ which corresponds to the symmetry algebra defined by (18) in case of an arbitrary function $\lambda(S)$.

If the function $\lambda(S)$ has a special form given by (20) we obtain a richer symmetry group. The solution 
to the system of equations (24) with the functions $\xi, \tau, \phi$ defined by (21) and initial conditions (27) have the form

$$
\begin{aligned}
\tilde{S} & =S e^{a_{1} \epsilon}, \quad \epsilon \in(-\infty, \infty), \\
\tilde{t} & =t+a_{2} \epsilon, \\
\tilde{u} & =u e^{a_{1}(1-k) \epsilon}+\frac{a_{3}}{a_{1} k} S \epsilon e^{a_{1} \epsilon}\left(1-e^{-a_{1} k \epsilon}\right) \\
& +\frac{a_{4}}{a_{1}(1-k)}\left(e^{a_{1}(1-k) \epsilon}-1\right), \quad k \neq 0, k \neq 1 \\
\tilde{u} & =u e^{a_{1} \epsilon}+a_{3} S \epsilon e^{a_{1} \epsilon}+\frac{a_{4}}{a_{1}}\left(e^{a_{1} \epsilon}-1\right), k=0, \\
\tilde{u} & =u+\frac{a_{3}}{a_{1}} S\left(e^{a_{1} \epsilon}-1\right)+a_{4} \epsilon, k=1,
\end{aligned}
$$

where we assume that $a_{1} \neq 0$ because the case $a_{1}=0$ coincides with the former case (28)-(30).

We will use the symmetry group $G_{\Delta}$ to construct invariant solutions to equation (4). To obtain the invariants of the symmetry group $G_{\Delta}$ we exclude $\epsilon$ from the equations (28)-(30) or in the special case from equations (31)-(33).

In the first case the symmetry group $G_{\Delta}$ is very poor and we can obtain just the following invariants

$$
\begin{aligned}
& i n v_{1}=S, \\
& i n v_{2}=u-\left(a_{3} S+a_{4}\right) / a_{2}, \quad a_{2} \neq 0 .
\end{aligned}
$$

These invariants are useless because they do not lead to any reduction of (4). form

In the special case (20) the symmetry group admits two functionally independent invariants of the

$$
\begin{aligned}
& i n v_{1}=\log S+a t, \quad a=a_{1} / a_{2}, a_{2} \neq 0 \\
& i n v_{2}=u S^{(k-1)} .
\end{aligned}
$$

In general the form of invariants is not unique because each function of invariants is an invariant. But it is possible to obtain just two non-trivial functionally independent invariants which we take in the form (35), (36). The invariants can be used as new independent and dependent variables in order to reduce the partial differential equation (4) with the special function $\lambda(S)$ defined by (20) to an ordinary differential equation.

3. The special case $\lambda(S)=\omega S^{k}$. Let us study a special case of equation (4) with $\lambda(S)=$ $\omega S^{k}, \omega, k \in R$. The equation under investigation is now

$$
u_{t}+\frac{\sigma^{2} S^{2}}{2} \frac{u_{S S}}{\left(1-b S^{k+1} u_{S S}\right)^{2}}=0
$$

with the constant $b=\rho \omega$. As usual we assume that $\rho \in(0,1)$. The value of the constant $\omega$ depends on the corresponding option type and in our investigation it can be assumed that $\omega$ is an arbitrary constant, $\omega \neq 0$. The variables $S, t$ are in the intervals

$$
S>0, \quad t \in[0, T], \quad T>0 .
$$

Remark 3.1. The case $b=0$, i.e. $\rho=0$ or $\omega=0$ leads to the well known linear Black-Scholes model and we will exclude this case from our investigations.

We will suppose that the denominator in equation (4) (correspondingly (37)) is non equal to zero identically.

Let us study the denominator in the second term of the equation (37). It will be equal to zero if the function $u(S, t)$ satisfies the equation

$$
1-b S^{k+1} u_{S S}=0 .
$$


The solution to this equation is a function $u_{0}(S, t)$

$$
\begin{aligned}
& u_{0}(S, t)=\frac{1}{b k(k-1)} S^{1-k}+S c_{1}(t)+c_{2}(t), \quad b \neq 0, k \neq 0,1, \\
& u_{0}(S, t)=-\frac{1}{b} \log S+S c_{1}(t)+c_{2}(t), \quad b \neq 0, k=1, \\
& u_{0}(S, t)=\frac{1}{b} S \log S+S c_{1}(t)+c_{2}(t), \quad b \neq 0, k=0,
\end{aligned}
$$

where the functions $c_{1}(t)$ and $c_{2}(t)$ are arbitrary functions of the variable $t$.

In the sequel we will assume that the denominator in the second term of the equation (37) is not identically zero, i.e., a solution $u(S, t)$ is not equal to the function $u_{0}(S, t)(40)$ except in a discrete set of points.

Let us now introduce new invariant variables

$$
\begin{aligned}
z & =\log S+a t, \quad a \in R, a \neq 0 \\
v & =u S^{(k-1)}
\end{aligned}
$$

After this substitution equation (37) will be reduced to an ordinary differential equation

$$
a v_{z}+\frac{\sigma^{2}}{2} \frac{v_{z z}+(1-2 k) v_{z}-k(1-k) v}{\left(1-b\left(v_{z z}+(1-2 k) v_{z}-k(1-k) v\right)\right)^{2}}=0, \quad a, b \neq 0
$$

Elementary solutions to this equation we obtain if we assume that $v=$ const. or $v_{z}=$ const. . It is easy to prove that there exists the trivial solution $v=0$ for any $k$ and $v_{z}=0$ if $k \neq 0,1$, and the solutions $v=$ const. $\neq 0, v_{z}=$ const. $\neq 0$ if $k=0,1$ only. The condition that the denominator in (42) is non equal to zero, i.e.,

$$
\left(1-b\left(v_{z z}+(1-2 k) v_{z}-k(1-k) v\right)\right)^{2} \neq 0
$$

corresponds to equation (39) in new variables $z, v$.

If the function $v(z)$ satisfies the inequality (43) then we can multiply both terms of equation (42) with the denominator of the second term. In equation (42) all coefficients are constants hence we can reduce the order of the equation. We assume that $v, v_{z} \neq$ const. We now choose as a new independent variable $v$ and introduce as a new dependent variable $x(v)=v_{z}(z)$. This variable substitution reduces equation (42) to a first order differential equation which is second order polynomial corresponding to the function $x(v)_{v}$. Under assumption (43) the set of solutions to equation (42) is equivalent to a union of solution sets of the following equations

$$
\begin{aligned}
x & =0, k=0,1 \\
x_{v} & =-1+2 k-\frac{\sigma^{2}}{4 a b^{2} x^{2}}+\frac{1}{b x}+\frac{k(1-k) v}{x}-\frac{\sqrt{\sigma^{2}\left(\sigma^{2}-8 a b x\right)}}{4 a b^{2} x^{2}}, \\
x_{v} & =-1+2 k-\frac{\sigma^{2}}{4 a b^{2} x^{2}}+\frac{1}{b x}+\frac{k(1-k) v}{x}+\frac{\sqrt{\sigma^{2}\left(\sigma^{2}-8 a b x\right)}}{4 a b^{2} x^{2}} .
\end{aligned}
$$

Equations (44) are of an autonomous type if the parameter $k$ is equal to $k=0,1$ only. We see that these are exactly the cases in which the corresponding Lie-algebra (23) has a two dimensional center. The case $k=0$ was studied earlier in [Bordag and Chmakova, 2007], [Chmakova, 2005] and in [Bordag and Frey, 2009]. In the next section we will study the case $k=1$.

4. The special case $\lambda=\omega S$. If we put $k=1$ in (20) then equation (42) takes the form

$$
v_{z}+q \frac{v_{z z}-v_{z}}{\left(1-b\left(v_{z z}-v_{z}\right)\right)^{2}}=0
$$


where $q=\frac{\sigma^{2}}{2 a}, a, b \neq 0$. It is an autonomous equation which possesses a simple structure. We will use this structure and introduce a more simple substitution as described at the end of the previous section to reduce the order of equation.

One family of solutions to this equation is very easy to find. We just suppose that the value $v_{z}(z)$ is equal to a constant. The equation (45) admits as a solution the value $v_{z}=(-1 \pm \sqrt{q}) / b$ consequently the corresponding solution $u(s, t)$ to $(37)$ with $\lambda=\omega S$ can be represented by the formula

$$
u(S, t)=\frac{1}{\rho \omega}(-1 \pm \sqrt{q})(\log S+a t)+c, a>0,
$$

where $c$ is an arbitrary constant.

To find other families of solutions we introduce a new dependent variable

$$
y(z)=v_{z}(z)
$$

and assume that the denominator of the equation (45) is not equal to zero, i.e.

$$
v(z) \neq-\frac{z}{b}+c_{1} e^{z}+c_{2}, \text { i.e. } y(z) \neq-\frac{1}{b}+c_{1} e^{z}
$$

where $c_{1}, c_{2}$ are arbitrary constants.

We multiply both terms of equation (45) by the denominator of the second term and obtain

$$
y y_{z}^{2}-2\left(y^{2}+\frac{1}{b} y-\frac{q}{2 b^{2}}\right) y_{z}+\left(y^{2}+\frac{2}{b} y+\left(\frac{1-q}{b^{2}}\right)\right) y=0, \quad b \neq 0 .
$$

We denote the left hand side of this equation by $F\left(y, y_{z}\right)$. The equation (49) can possess exceptional solutions which are the solutions to a system

$$
\frac{\partial F\left(y, y_{z}\right)}{\partial y_{z}}=0, \quad F\left(y, y_{z}\right)=0
$$

The first equation in this system defines a discriminant curve which has the form

$$
y(z)=\frac{q}{4 b} .
$$

If this curve is also a solution to the original equation (49) then we obtain an exceptional solution. We obtain an exceptional solution if $q=4$, i.e. $a=\sigma^{2} / 8$. It has the form

$$
y(z)=\frac{1}{b}
$$

This solution belongs to the family of solutions (54) by the specified value of the parameter $q$. In all other cases the equation (49) does not possess any exceptional solutions.

Hence the set of solutions to equation (49) is a union of solution sets of following equations

$$
\begin{aligned}
y & =0, \\
y & =(-1 \pm \sqrt{q}) / b, \\
y_{z} & =\left(y^{2}+\frac{1}{b} y-\frac{q}{2 b^{2}}-\sqrt{\frac{\sigma^{2}}{2 a b^{3}}\left(\frac{q}{4 b}-y\right)}\right) \frac{1}{y}, y \neq 0, \\
y_{z} & =\left(y^{2}+\frac{1}{b} y-\frac{q}{2 b^{2}}+\sqrt{\frac{q}{b^{3}}\left(\frac{q}{4 b}-y\right)}\right) \frac{1}{y}, y \neq 0,
\end{aligned}
$$

where one of the solutions (54) is an exceptional solution (52) by $q=4$. We denote the right hand side of equations (55), (56) by $f(y)$. The Lipschitz condition for equations of type $y_{z}=f(y)$ is satisfied in 
all points where the derivative $\frac{\partial f}{\partial y}$ exists and is bounded. It is easy to see that this condition will not be satisfied by

$$
y=0, \quad y=\frac{q}{4 b}, \quad y=\infty .
$$

It means that on the lines (57) the uniqueness of solutions to equations (55), (56) can be lost. We will study in detail the behavior of solutions in the neighborhood of lines (57). For this purpose we look at the equation (49) from another point of view. If we assume now that $z, y, y_{z}$ are complex variables and denote

$$
y(z)=\zeta, \quad y_{z}(z)=w, \quad \zeta, w \in C,
$$

then the equation (49) takes the form

$$
F(\zeta, w)=\zeta w^{2}-2\left(\zeta^{2}+\frac{1}{b} \zeta-\frac{q}{2 b^{2}}\right) w+\left(\zeta^{2}+\frac{2}{b} \zeta+\frac{1-q}{b^{2}}\right) \zeta=0,
$$

where $b \neq 0$. The equation (59) is an algebraic relation in $C^{2}$ and defines a plane curve in this space. The polynomial $F(\zeta, w)$ is an irreducible polynomial if at all roots $w_{r}(z)$ of $F\left(\zeta, w_{r}\right)$ either the partial derivative $F_{\zeta}\left(\zeta, w_{r}\right)$ or $F_{w}\left(\zeta, w_{r}\right)$ are non equal to zero. It is easy to prove that the polynomial (59) is irreducible.

We can treat equation (59) as an algebraic relation which defines a Riemann surface $\Gamma: F(\zeta, w)=0$ of $w=w(\zeta)$ as a compact manifold over the $\zeta$-sphere. The function $w(\zeta)$ is uniquely analytically extended over the Riemann surface $\Gamma$ of two sheets over the $\zeta$-sphere. We find all singular or branch points of $w(\zeta)$ if we study the roots of the first coefficient of the polynomial $F(\zeta, w)$, the common roots of equations

$$
F(\zeta, w)=0, \quad F_{w}(\zeta, w)=0, \quad \zeta, w \in C \cup \infty
$$

and the point $\zeta=\infty$. The set of singular or branch points consists of the points

$$
\zeta_{1}=0, \quad \zeta_{2}=\frac{q}{4 b}, \quad \zeta_{3}=\infty .
$$

As expected we got the same set of points as in real case (57) by the study of the Lipschitz condition but now the behavior of solutions at the points is more visible.

The points $\zeta_{2}, \zeta_{3}$ are the branch points at which two sheets of $\Gamma$ are glued on. We remark that

$$
w\left(\zeta_{2}\right)=\frac{1}{b}(q-4)+t \frac{1}{4 \sqrt{-b q}}+\cdots, \quad t^{2}=\zeta-\frac{q}{4 b},
$$

where $t$ is a local parameter in the neighborhood of $\zeta_{2}$. For the special value of $q=4$ the value $w\left(\zeta_{2}\right)$ is equal to zero.

At the point $\zeta_{3}=\infty$ we have

$$
w(\zeta)=\frac{1}{t^{2}}+\frac{1}{b}+t \sqrt{\frac{-q}{4 b^{3}}}, \quad t^{2}=\frac{1}{\zeta}, \quad \zeta \rightarrow \infty,
$$

where $t$ is a local parameter in the neighborhood of $\zeta_{3}$. At the point $\zeta_{1}=0$ the function $w(\zeta)$ has the following behavior

$$
\begin{aligned}
& w(\zeta) \sim-\frac{q}{b^{2}} \frac{1}{\zeta}, \zeta \rightarrow \zeta_{1}=0, \text { on the principal sheet, } \\
& w(\zeta) \sim(1-q) \zeta, \zeta \rightarrow \zeta_{1}=0, q \neq 1, \quad \text { on the second sheet, } \\
& w(\zeta) \sim-2 b^{2} \zeta^{2}, \zeta \rightarrow \zeta_{1}=0, q=1, \quad \text { on the second sheet. }
\end{aligned}
$$

Any solution $w(\zeta)$ to an irreducible algebraic equation (59) is meromorphic on this compact Riemann surface $\Gamma$ of the genus 0 and has a pole of the order one correspondingly (63) over the point $\zeta_{1}=0$ and the pole of the second order over $\zeta_{3}=\infty$. It means also that the meromorphic function $w(\zeta)$ cannot be defined on a manifold of less than 2 sheets over the $\zeta$ sphere. 
To solve differential equations (55) and (56) from this point of view it is equivalent to integrate on $\Gamma$ a differential of type $\frac{\mathrm{d} \zeta}{w(\zeta)}$ and then to solve an Abel's inverse problem of degenerated type

$$
\int \frac{\mathrm{d} \zeta}{w(\zeta)}=z+\text { const. }
$$

The integration can be done very easily because we can introduce a uniformizing parameter on the Riemann surface $\Gamma$ and represent the integral (66) in terms of rational functions merged possibly with logarithmic terms.

To realize this program we introduce a new variable (our uniformizing parameter $p$ ) in the way

$$
\begin{aligned}
\zeta & =\frac{q\left(1-p^{2}\right)}{4 b} \\
w & =\frac{(1-p)\left(q(1+p)^{2}-4\right)}{4 b(p+1)} .
\end{aligned}
$$

Then the equations (55) and (56) will take the form

$$
\begin{aligned}
& 2 q \int \frac{p(p+1) \mathrm{d} p}{(p-1)\left(q(p+1)^{2}-4\right)}=z+\text { const, } \\
& 2 q \int \frac{p(p-1) \mathrm{d} p}{(p+1)\left(q(p-1)^{2}-4\right)}=z+\text { const. }
\end{aligned}
$$

The integration procedure of equation (69) gives rise to the following relations

$$
\begin{aligned}
& 2 q \log (p-1)+(q-\sqrt{q}-2) \log ((p+1) \sqrt{q}-2) \\
& +(q+\sqrt{q}-2) \log ((p+1) \sqrt{q}+2)=2(q-1) z+c, \quad q \neq 1, q>0 \\
& \frac{1}{1-p}+\frac{1}{4} \log \frac{(p+3)^{3}}{(p-1)^{5}}=z+c, \quad q=1 \\
& 2 \sqrt{(-q)} \arctan ((p+1) \sqrt{(-q)} / 2)-2 q \log (p-1) \\
& +(2-q) \log \left(4-q(p+1)^{2}\right)=2(1-q) z+c, \quad q<0
\end{aligned}
$$

where $c$ is an arbitrary constant. The equation (70) leads to

$$
\begin{aligned}
& 2 q \log (p+1)+(q+\sqrt{q}-2) \log ((p-1) \sqrt{q}-2) \\
& +(q-\sqrt{q}-2) \log ((p-1) \sqrt{q}+2)=2(q-1) z+c, \quad q \neq 1, q>0 \\
& \frac{1}{p+1}+\frac{1}{4} \log \frac{(p-3)^{3}}{(p+1)^{5}}=z+c, \quad q=1 \\
& -2 \sqrt{(-q)} \arctan ((p-1) \sqrt{(-q)} / 2)-2 q \log (1+p) \\
& +(2-q) \log \left(4-q(p-1)^{2}\right)=2(1-q) z+c, \quad q<0
\end{aligned}
$$

where $c$ is an arbitrary constant.

The relations (71)-(76) are first order ordinary differential equations because of the substitutions (58) and (47) we have

$$
p=\sqrt{1-\frac{4 b}{q} v_{z}} .
$$

All these results can be collected to the following theorem.

Theorem 4.1. The equation (45) for arbitrary values of the parameters $q, b \neq 0$ can be reduced to the set of first order differential equations which consists of the equations

$$
v_{z}=0, \quad v_{z}=(-1 \pm \sqrt{q}) / b
$$

and equations (71)-(76). The complete set of solutions to the equation (45) coincides with the union of solutions to these equations. 
To solve equations (71)-(76) exactly we should first invert these formulas in order to obtain an exact representation $p$ as a function of $z$. If an exact formula for the function $p=p(z)$ is found we can use the substitution (77) to obtain an explicit ordinary differential equation of the type $v_{z}(z)=f(z)$ or another suitable type and if it possible then to integrate this equation.

But even in the first step we would not be able to do this for an arbitrary value of the parameter $q$. It means we have just implicit representations for the solutions to the equation (45) as solutions to the implicit first order differential equations (71)-(76).

\subsection{Exact invariant solutions in case of a fixed relation}

between variables $S$ and $t$. For a special value of the parameter $q$ we can invert the equations (71) and (74). Let us take $q=4$, i.e., the relation between variables $S, t$ is fixed in the form

$$
z=\log S+\frac{\sigma^{2}}{8} t .
$$

In this case the equation (71) takes the form

$$
(p-1)^{2}(p+2)=2 c \exp (3 z / 2)
$$

and correspondingly the equation (74) the form

$$
(p+1)^{2}(p-2)=2 c \exp (3 z / 2),
$$

where $c$ is an arbitrary constant. It is easy to see that the equations (80) and (81) are connected by a transformation

$$
p \rightarrow-p, \quad c \rightarrow-c .
$$

This symmetry arises from the symmetry of the underlining Riemann surface $\Gamma$ (59) and corresponds to a change of the sheets on $\Gamma$.

Theorem 4.2. The second order differential equation

$$
v_{z}+4 \frac{v_{z z}-v_{z}}{\left(1-b\left(v_{z z}-v_{z}\right)\right)^{2}}=0,
$$

is exactly integrable for an arbitrary value of the parameter $b$. The complete set of solutions for $b \neq 0$ is given by the union of solutions (87), (89) -(92) and solutions

$$
v(z)=d, \quad v(z)=-\frac{3}{b} z+d, \quad v(z)=\frac{1}{b} z+d,
$$

where $d$ is an arbitrary constant. The last solution in (84) corresponds to the exceptional solution to equation (49).

For $b=0$ equation (83) is linear and its solutions are given by $v(z)=d_{1}+d_{2} \exp (3 z / 4)$, where $d_{1}, d_{2}$ are arbitrary constants.

Proof. Because of the symmetry (82) it is sufficient to study either the equations (80) or (81) for $c \in R$ or both these equations for $c>0$. The value $c=0$ can be excluded because it complies with the constant value of $p(z)$ and correspondingly constant value of $v_{z}(z)$, but all such cases are studied before and the solutions are given by $(84)$.

We will study equation (81) in case $c \in R \backslash\{0\}$ and obtain on this way the complete class of exact solutions for equations (80)-(81).

Equation (81) for $c>0$ has a one real root only. It leads to an ordinary differential equation of the form

$$
\begin{aligned}
v_{z}(z)= & -\frac{1}{b}\left(1+\left(1+c e^{\frac{3 z}{2}}+\sqrt{2 c e^{\frac{3 z}{2}}+c^{2} e^{3 z}}\right)^{-\frac{2}{3}}\right. \\
& \left.+\left(1+c e^{\frac{3 z}{2}}+\sqrt{2 c e^{\frac{3 z}{2}}+c^{2} e^{3 z}}\right)^{\frac{2}{3}}\right), \quad c>0 .
\end{aligned}
$$


Equation (85) can be exactly integrated if we use an Euler substitution and introduce a new independent variable

$$
\tau=2\left(1+c e^{\frac{3 z}{2}}+\sqrt{2 c e^{\frac{3 z}{2}}+c^{2} e^{3 z}}\right) .
$$

The corresponding solution is given by

$$
\begin{aligned}
& v_{r}(z)=-\frac{1}{b}\left(\left(1+c e^{\frac{3 z}{2}}+\sqrt{2 c e^{\frac{3 z}{2}}+c^{2} e^{3 z}}\right)^{-\frac{2}{3}}+\left(1+c e^{\frac{3 z}{2}}+\sqrt{2 c e^{\frac{3 z}{2}}+c^{2} e^{3 z}}\right)^{\frac{2}{3}}\right. \\
& \left.+2 \log \left(\left(1+c e^{\frac{3 z}{2}}+\sqrt{2 c e^{\frac{3 z}{2}}+c^{2} e^{3 z}}\right)^{-\frac{1}{3}}+\left(1+c e^{\frac{3 z z}{2}}+\sqrt{2 c e^{\frac{3 z}{2}}+c^{2} e^{3 z}}\right)^{\frac{1}{3}}-2\right)\right) \\
& +d,
\end{aligned}
$$

where $d \in R$ is an arbitrary constant.

If in the right hand side of equation (81) the parameter $c$ satisfies the inequality $c<0$ and the variable $z$ chosen in the region

$$
z \in\left(-\infty,-\frac{4}{3} \ln |c|\right)
$$

then the equation on $p$ possesses maximal three real roots.

These three roots of cubic equation (81) give rise to three differential equations of type $v_{z}=(1-$ $\left.p^{2}(z)\right) / b$. The equations can be exactly solved and we find correspondingly three solutions $v_{i}(z), i=$ $1,2,3$.

The first solution is given by the expression

$$
\begin{aligned}
v_{1}(z) & =\frac{z}{b}-\frac{2}{b} \cos \left(\frac{2}{3} \arccos \left(1-|c| e^{\frac{3 z}{2}}\right)\right) \\
& -\frac{4}{3 b} \log \left(1+2 \cos \left(\frac{1}{3} \arccos \left(1-|c| e^{\frac{3 z}{2}}\right)\right)\right) \\
& -\frac{16}{3 b} \log \left(\sin \left(\frac{1}{6} \arccos \left(1-|c| e^{\frac{3 z}{2}}\right)\right)\right)+d,
\end{aligned}
$$

where $d \in R$ is an arbitrary constant. The second solution is given by the formula

$$
\begin{aligned}
v_{2}(z) & =\frac{z}{b}-\frac{2}{b} \cos \left(\frac{2}{3} \pi+\frac{2}{3} \arccos \left(-1+|c| e^{\frac{3 z}{2}}\right)\right) \\
& -\frac{4}{3 b} \log \left(1+2 \cos \left(\frac{1}{3} \pi+\frac{1}{3} \arccos \left(-1+|c| e^{\frac{3 z}{2}}\right)\right)\right) \\
& -\frac{16}{3 b} \log \left(\sin \left(\frac{1}{6} \pi+\frac{1}{6} \arccos \left(-1+|c| e^{\frac{3 z}{2}}\right)\right)\right)+d
\end{aligned}
$$

where $d \in R$ is an arbitrary constant. The first and second solutions are defined up to the point $z=-\frac{4}{3} \ln |c|$ where they coincide (see Fig. 1).

The third solution for $z<-\frac{4}{3} \ln |c|$ is given by the formula

$$
\begin{aligned}
v_{3,1}(z) & =\frac{z}{b}-\frac{2}{b} \cos \left(\frac{2}{3} \arccos \left(-1+|c| e^{\frac{3 z}{2}}\right)\right) \\
& -\frac{4}{3 b} \log \left(-1+2 \cos \left(\frac{1}{3} \arccos \left(-1+|c| e^{\frac{3 z}{2}}\right)\right)\right) \\
& -\frac{16}{3 b} \log \left(\cos \left(\frac{1}{6} \arccos \left(-1+|c| e^{\frac{3 z}{2}}\right)\right)\right)+d,
\end{aligned}
$$


where $d \in R$ is an arbitrary constant. In case $z>-\frac{4}{3} \ln |c|$ the polynomial (81) has a one real root and the corresponding solution can be represented by the formula

$$
\begin{aligned}
v_{3,2}(z) & =\frac{z}{b}-\frac{2}{b} \cosh \left(\frac{2}{3} \operatorname{arccosh}\left(-1+|c| e^{\frac{3 z}{2}}\right)\right) \\
& -\frac{16}{3 b} \log \left(\cosh \left(\frac{1}{6} \operatorname{arccosh}\left(-1+|c| e^{\frac{3 z}{2}}\right)\right)\right) \\
& -\frac{4}{3 b} \log \left(-1+2 \cosh \left(\frac{1}{3} \operatorname{arccosh}\left(-1+|c| e^{\frac{3 z}{2}}\right)\right)\right)+d
\end{aligned}
$$

The third solution is represented by formulas $v_{3,2}(z)$ and $v_{3,1}(z)$ for different values of the variable $z$.

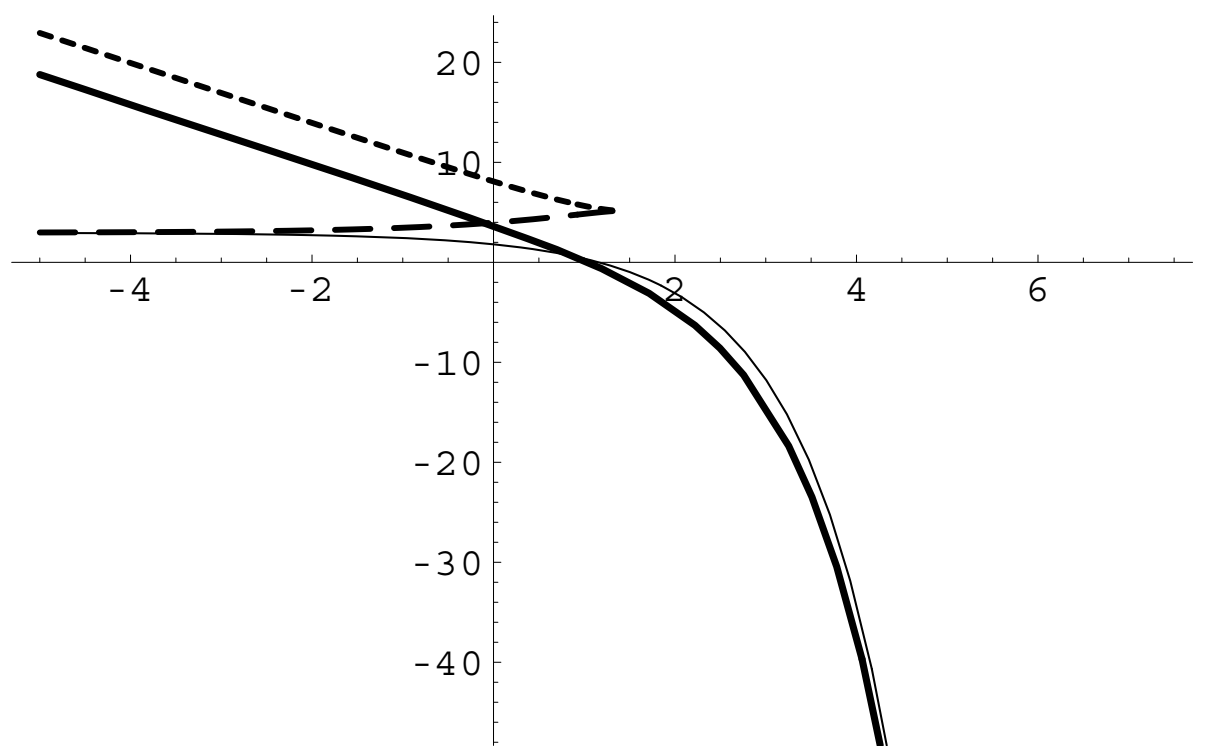

Figure 1: Plot of the solution $v_{r}(z),(87)$, (thick solid line), $v_{1}(z),(89)$, (short dashed line), $v_{2}(z)$, (90), (long dashed line) and the third solution $v_{3,1}(z), v_{3,2}(z),(91),(92)$, which is represented by the thin solid line. The parameters take the values $|c|=0.5, q=4, d=0, b=1$ and the variable $z \in(-5,4.5)$.

One of the sets of solutions (87), (89) -(92) for fixed parameters $b, c, d$ is represented in Fig. 1 . The solution $v_{r}(z)(87)$ and the third solution given by both (91) and (92) are defined for any values of $z$. The solutions $v_{1}(z)$ and $v_{2}(z)$ cannot be continued after the point $z=-\frac{4}{3} \ln |c|$ where they coincide.

If we keep in mind that $z=\log S+\frac{\sigma^{2}}{8} t$ and $v(z)=u(S, t)$ we can represent exact invariant solutions to equation (37). The solution (87) gives rise to an invariant solution $u_{r}(S, t)$ in the form

$$
\begin{aligned}
u_{r}(S, t)= & -\frac{1}{\omega \rho}\left(1+c S^{\frac{3}{2}} e^{\frac{3 \sigma^{2}}{16} t}+\sqrt{2 c S^{\frac{3}{2}} e^{\frac{3 \sigma^{2}}{16} t}+c^{2} S^{3} e^{\frac{3 \sigma^{2}}{8} t}}\right)^{-\frac{2}{3}} \\
& -\frac{1}{\omega \rho}\left(1+c S^{\frac{3}{2}} e^{\frac{3 \sigma^{2}}{16} t}+\sqrt{2 c S^{\frac{3}{2}} e^{\frac{3 \sigma^{2}}{16} t}+c^{2} S^{3} e^{\frac{3 \sigma^{2}}{8} t}}\right)^{\frac{2}{3}} \\
- & \frac{2}{\omega \rho} \log \left(\left(1+c S^{\frac{3}{2}} e^{\frac{3 \sigma^{2}}{16} t}+\sqrt{2 c S^{\frac{3}{2}} e^{\frac{3 \sigma^{2}}{16} t}+c^{2} S^{3} e^{\frac{3 \sigma^{2}}{8} t}}\right)^{-\frac{1}{3}}\right.
\end{aligned}
$$




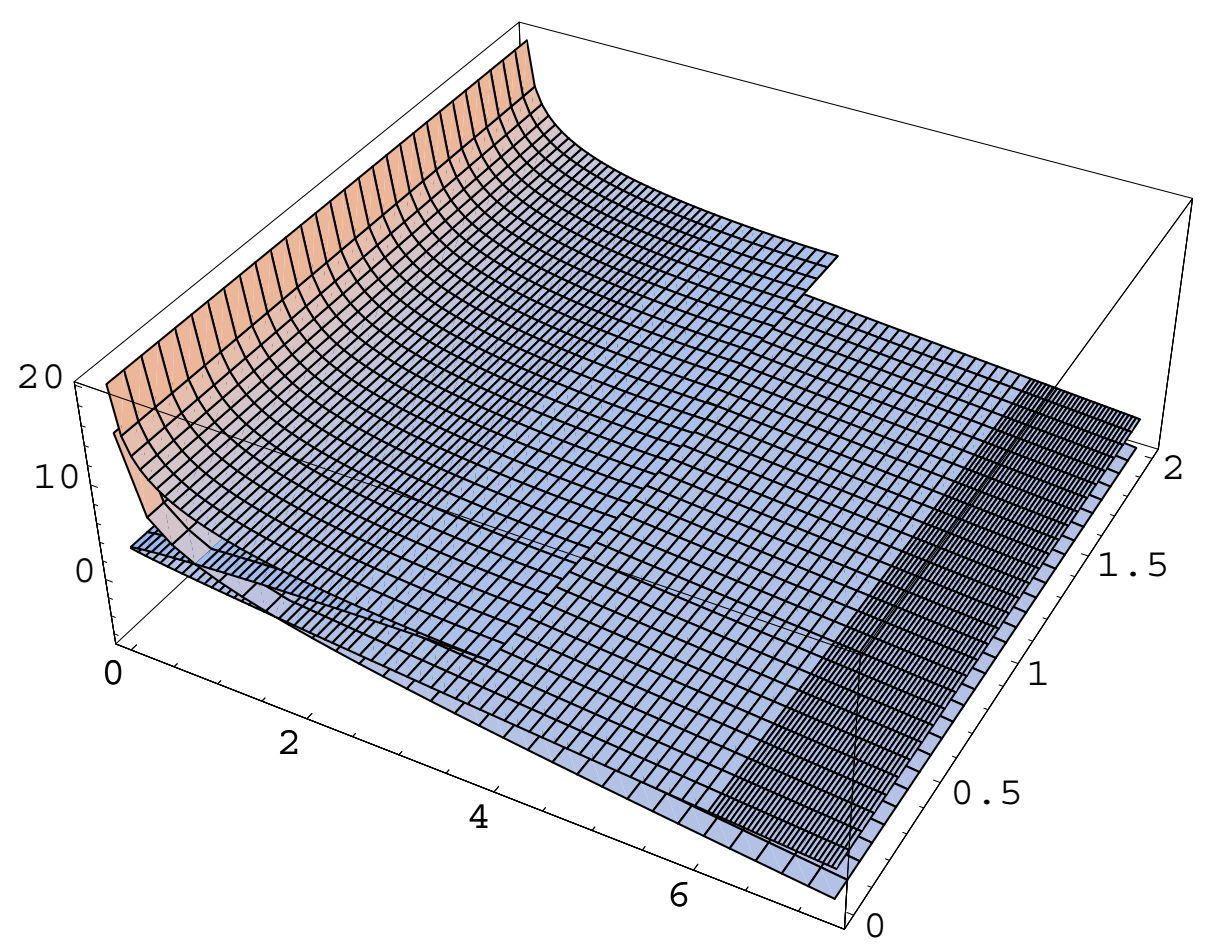

Figure 2: Plot of solutions $u_{r}(S, t), u_{1}(S, t), u_{2}(S, t), u_{3,1}(S, t), u_{3,2}(S, t)$ for the parameters $|c|=0.25, q=4, b=1.0, d=0$. The variables $S, t$ lie in intervals $S \in(0,9)$ and $t \in[0,2.0]$. All invariant solutions change slowly in $t$-direction.

$$
\left.+\left(1+c S^{\frac{3}{2}} e^{\frac{3 \sigma^{2}}{16} t}+\sqrt{2 c S^{\frac{3}{2}} e^{\frac{3 \sigma^{2}}{16} t}+c^{2} S^{3} e^{\frac{3 \sigma^{2}}{8} t}}\right)^{\frac{1}{3}}-2\right)+d
$$

where $d \in R, c>0$.

In case $c<0$ we can obtain correspondingly three solutions if

$$
0<S \leq|c|^{-\frac{4}{3}} \exp \left(-\frac{\sigma^{2}}{8} t\right) .
$$

The first solution is represented by

$$
\begin{aligned}
u_{1}(S, t) & =\frac{1}{\omega \rho}\left(\log S+\frac{\sigma^{2}}{8} t\right)-\frac{2}{\omega \rho} \cos \left(\frac{2}{3} \arccos \left(1-|c| S^{\frac{3}{2}} e^{\frac{3 \sigma^{2}}{16} t}\right)\right) \\
& -\frac{4}{3 \omega \rho} \log \left(1+2 \cos \left(\frac{1}{3} \arccos \left(1-|c| S^{\frac{3}{2}} e^{\frac{3 \sigma^{2}}{16} t}\right)\right)\right) \\
& -\frac{16}{3 \omega \rho} \log \left(\sin \left(\frac{1}{6} \arccos \left(1-|c| S^{\frac{3}{2}} e^{\frac{3 \sigma^{2}}{16} t}\right)\right)\right)+d
\end{aligned}
$$

where $d \in R, c<0$. The second solution is given by the formula

$$
\begin{aligned}
u_{2}(S, t) & =\frac{1}{\omega \rho}\left(\log S+\frac{\sigma^{2}}{8} t\right)-\frac{2}{\omega \rho} \cos \left(\frac{2}{3} \pi+\frac{2}{3} \arccos \left(-1+|c| S^{\frac{3}{2}} e^{\frac{3 \sigma^{2}}{16} t}\right)\right) \\
& -\frac{4}{3 \omega \rho} \log \left(1+2 \cos \left(\frac{1}{3} \pi+\frac{1}{3} \arccos \left(-1+|c| S^{\frac{3}{2}} e^{\frac{3 \sigma^{2}}{16} t}\right)\right)\right) \\
& -\frac{16}{3 \omega \rho} \log \left(\sin \left(\frac{1}{6} \pi+\frac{1}{6} \arccos \left(-1+|c| S^{\frac{3}{2}} e^{\frac{3 \sigma^{2}}{16} t}\right)\right)\right)+d,
\end{aligned}
$$


where $d \in R, c<0$. The first and second solutions are defined for the variables under conditions (94). They coincide along the curve

$$
S=|c|^{-4 / 3} \exp \left(-\frac{\sigma^{2}}{8} t\right)
$$

and cannot be continued further.

The third solution is defined by

$$
\begin{aligned}
u_{3,1}(S, t) & =\frac{1}{\omega \rho}\left(\log S+\frac{\sigma^{2}}{8} t\right)-\frac{2}{\omega \rho} \cos \left(\frac{2}{3} \arccos \left(-1+|c| S^{\frac{3}{2}} e^{\frac{3 \sigma^{2}}{16} t}\right)\right) \\
& -\frac{4}{3 \omega \rho} \log \left(-1+2 \cos \left(\frac{1}{3} \arccos \left(-1+|c| S^{\frac{3}{2}} e^{\frac{3 \sigma^{2}}{16} t}\right)\right)\right) \\
& -\frac{16}{3 \omega \rho} \log \left(\cos \left(\frac{1}{6} \arccos \left(-1+|c| S^{\frac{3}{2}} e^{\frac{3 \sigma^{2}}{16} t}\right)\right)\right)+d,
\end{aligned}
$$

where $d \in R$ and $S, t$ satisfied the condition (94).

In case $\log S+\frac{\sigma^{2}}{8} t>-\frac{4}{3} \ln |c|$ the third solution can be represented by the formula

$$
\begin{aligned}
u_{3,2}(S, t) & =\frac{1}{\omega \rho}\left(\log S+\frac{\sigma^{2}}{8} t\right)-\frac{2}{\omega \rho} \cosh \left(\frac{2}{3} \operatorname{arccosh}\left(-1+|c| S^{\frac{3}{2}} e^{\frac{3 \sigma^{2}}{16} t}\right)\right) \\
& -\frac{16}{3 \omega \rho} \log \left(\cosh \left(\frac{1}{6} \operatorname{arccosh}\left(-1+|c| S^{\frac{3}{2}} e^{\frac{3 \sigma^{2}}{16} t}\right)\right)\right) \\
& -\frac{4}{3 \omega \rho} \log \left(-1+2 \cosh \left(\frac{1}{3} \operatorname{arccosh}\left(-1+|c| S^{\frac{3}{2}} e^{\frac{3 \sigma^{2}}{16} t}\right)\right)\right)+d .
\end{aligned}
$$

The solution $u_{r}(S, t)(93)$ and the third solution given by $u_{3,1}, u_{3,2}(97),(98)$ are defined for all values of variables $t$ and $S>0$. They have a common intersection curve of type $S=$ const. $\exp \left(-\frac{\sigma^{2}}{8} t\right)$. The typical behavior of all these invariant solutions is represented in Fig. 2.

It should be noted that because of the symmetry properties (see Theorem 2.3.) any solution remains a solution if we add to each solution a linear function of $S$,

$$
u(S, t) \rightarrow u(S, t)+d_{1} S+d_{2},
$$

with arbitrary constants $d_{1}, d_{2}$.

It means we have two additional constants to model boundary and terminal conditions.

We first study the non-trivial solutions, i.e., $u(S, t) \neq d_{1} S+d_{2}$.

Previous results can be summed up in the following theorem describing the set of non-trivial invariant solutions to equation (4).

Theorem 4.3. $\quad$ 1. The equation (4) possesses non-trivial invariant solutions for the only special form of the function $\lambda(S)$ given by (20).

2. In case (20) the invariant solutions to equation (4) are defined by ordinary differential equations (44). In special cases $k=0,1$ equations (44) are of an autonomous type.

3. If $\lambda(S)=\omega S$, i.e. $k=1$, then the invariant solutions to equation (4) can be defined by the set of first order ordinary differential equations (71)-(76) and equation (78).

If additionally the parameter $q=4$, or equivalent in the first invariant (35) we chose $a=\sigma^{2} / 8$ then the complete set of invariant solutions (4) can be found exactly. This set of invariant solutions is given by formulas (93)-(98) and by solutions

$$
u(S, t)=d, \quad u(S, t)=-3 / b\left(\log S+\sigma^{2} t / 8\right), \quad u(S, t)=1 / b\left(\log S+\sigma^{2} t / 8\right),
$$

where $d$ is an arbitrary constant. This set of invariant solutions is unique up to the transformations of the symmetry group $G_{\Delta}$ given by theorem 2.3. 
5. Properties of invariant solutions. All solutions (93)-(98) have the form $u(S, t)=w(S, t) /(\omega \rho)$, where $w(S, t)$ is a smooth function of $S, t$. It means that the function $w(S, t)$ solves the equation (37) with $b=\omega \rho=1$, i.e.

$$
w_{t}+\frac{\sigma^{2} S^{2}}{2} \frac{w_{S S}}{\left(1-S^{2} w_{S S}\right)^{2}}=0 .
$$

In other words, if we find any solution to the above equation (100) for any fixed boundary and terminal conditions, we can immediately obtain the corresponding solution to the equation (37) if we just divide the function $w(S, t)$ by $b=\omega \rho$ (the solution $u(S, t)$ satisfies the boundary and terminal conditions which we obtain if just divide the corresponding conditions on the function $w(S, t)$ by $b$ ). Therefor any $\rho$-dependence of a solution to (37) is trivial. It means as well that if the terminal conditions are fixed $u(S, T)=h(S)$ then the value $u(S, t)$ will increase if the value of the parameter $\rho$ increases. This dependence of hedge costs on the position of the large trader on market is very natural.

If the parameter $\rho \rightarrow 0$ then equation (4) and correspondingly equation (37) will be reduced to the linear Black-Scholes equation but solutions (93)-(98) which we obtained here will be completely blown up by $\rho \rightarrow 0$ because of the factor $1 / b=1 /(\omega \rho)$ in the formulas (93)-(98). It means that the solutions $u(S, t)(93), u_{1}(S, t)(95), u_{2}(S, t)(96), u_{3,1}(S, t)(97), u_{3,2}(S, t)(98)$, have no one counterpart in a linear case.

This phenomena is rather typical for nonlinear partial differential equations with singular perturbations and was described as well in [Bordag and Chmakova, 2007], [Chmakova, 2005],

[Bordag and Frey, 2009] for the invariant solutions to equation (37) with $k=0$.

The families of exact solutions reflect the nonlinearity of this equation in a essential way. If we take a numerical method which was developed for the linear Black-Scholes equation or other types of parabolic equations and will test it for a new type of a nonlinear equation we should take, if possible, the solutions which reflect this nonlinearity in the most complete way. The existence of non-trivial explicit solutions allows to test different numerical methods usually used to calculate hedge-costs of derivatives (see [Bordag and Chmakova, 2007]).

We obtain a typical terminal payoff function for the solutions (93)-(98) if we just fix $t=T$. All these payoffs are smooth functions. Other sides a typical payoff of derivatives similar to combination of calls and puts is a continuous piece-wise linear function. The smooth payoffs are more convenient for numerical calculations and usually one replaced the standard payoffs by the corresponding solution to the Black-Scholes model for a very small time interval. If also in that case the numerical method does not work properly, there is no hope that it works better in a worse case and the fact that the payoffs are smooth functions is immaterial for these purposes.

In order to illustrate how we can model some typical payoffs we investigate asymptotic properties of solutions (93)-(98) as $S \rightarrow 0$ and as $S \rightarrow \infty$.

Using the exact formulas for solutions we retain the first two terms and obtain as $S \rightarrow 0$

$$
\begin{aligned}
u_{1}(S, t) & \sim-\frac{1}{b}\left(2+\frac{4}{3} \log \left(|c|^{2} 2^{-2} 3^{-3}\right)+\frac{3}{8} \sigma^{2} t+3 \log S+\mathcal{O}\left(S^{3 / 2}\right)\right), \\
u_{2}(S, t) & \sim \frac{1}{b}\left(1+\frac{1}{3} \log \left(2^{8} 3^{-6}|c|^{-2}\right)+\frac{2^{3} \sqrt{2|c|}}{3^{4 / 3}} e^{\frac{3}{32} \sigma^{2} t} S^{3 / 4}+\mathcal{O}\left(S^{3 / 2}\right)\right), \\
u_{3,1}(S, t) & \sim \frac{1}{b}\left(1+\frac{1}{3} \log \left(2^{8} 3^{-6}|c|^{-2}\right)-\frac{2^{3} \sqrt{2|c|}}{3^{4 / 3}} e^{\frac{3}{32} \sigma^{2} t} S^{3 / 4}+\mathcal{O}\left(S^{3 / 2}\right)\right), \\
u_{r}(S, t) & \sim-\frac{1}{b}\left(2+2 \log \left(23^{-2} c\right)+\frac{3}{8} \sigma^{2} t+3 \log S+\mathcal{O}\left(S^{5 / 4}\right)\right) .
\end{aligned}
$$

In Fig. 5 we present solutions (93)-(98) in a case where both additional constants $d_{1}=d_{2}$ in (99) are equal to zero, i.e. without linear background. 
Plot of solutions (93)-(98) by $\sigma=0.4, b=1,|c|=0.5, d_{1}=0, d_{2}=0$.

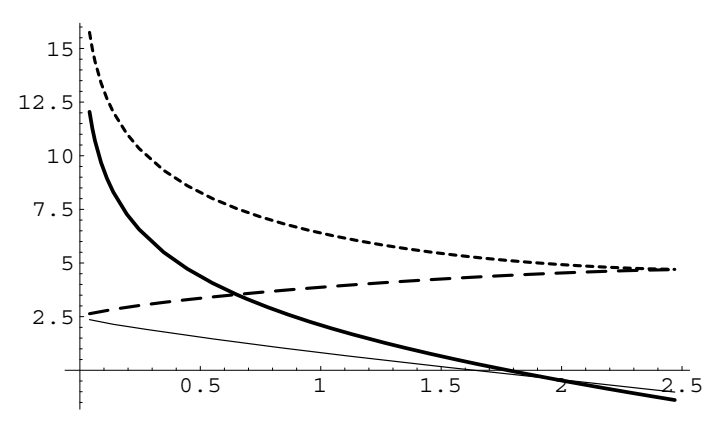

Figure 3: Behavior of solutions $u_{r}(z)$, (93), (thick solid line), $u_{1}(z)$, (95), (short dashed line), $u_{2}(z)$, (96), (long dashed line) and $u_{3,1}(z),(97)$ (thin solid line) in the neighborhood of $S \sim 0$, for $t=0$, where $S$ in (94).

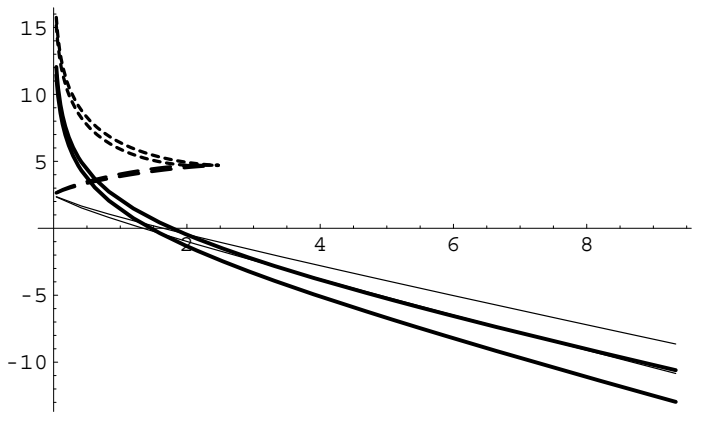

Figure 4: The same solutions for $t=0$. and $t=10$. for $S \in(0.04,9], u_{r}(z),(93)$, (thick solid line), $u_{1}(z)$, (95), (short dashed line), $u_{2}(z)$, (96), (long dashed line) and $u_{3,1}(z), u_{3,2}(z),(97),(98)$ (thin solid line).

If $S$ is large enough we have just two solutions. The asymptotic behavior both solutions $u_{r}(S, t),(93)$, and $u_{3,2},(98)$, coincides in the main terms as $S \rightarrow \infty$ and is given by formula

$$
u_{r}(S, t), u_{3,2}(S, t) \sim-\frac{1}{b}\left((2|c|)^{2 / 3} e^{\frac{3 \sigma^{2}}{8} t} S+\log S+\mathcal{O}(1)\right), \quad S \rightarrow \infty .
$$

The main term in formulas (101)-(105) depends on the time and on the constant $c$.

The families of exact solutions (93)-(98) have the following parameters $\omega, c, d_{1}, d_{2}$ which can be used to match suitable boundary and terminal conditions to a desired accuracy. The formulas (101)-(105) can be useful for these purposes. In Fig. 5 we represent as an example a long strip payoff and the solution $u_{r}(S, t),(93)$, which partly matches this payoff.

6. Acknowledgments. The author is grateful to Albert N. Shiryaev for the interesting and fruitful discussions.

\section{References}

[Bordag, 2005] Bordag, L. A. (2005). New types of solutions for nonlinear Black-Scholes model. Abstracts of the twelfth General Meeting of EWM. Volgograd, September 18-24, 2005.

[Bordag, 2006] Bordag, L. A. (2006). Symmetry reductions of a nonlinear model of financial derivatives. arXiv:math.AP/0604207, 09.04.2006.

[Bordag and Chmakova, 2007] Bordag, L. A. and Chmakova, A. Y. (2007). Explicit solutions for a nonlinear model of financial derivatives. International Journal of Theoretical and Applied Finance, $10: 1-21$.

[Bordag and Frey, 2009] Bordag, L. A. and Frey, R. (2009). Nonlinear option pricing models for illiquid markets: scaling properties and explicit solutions. Chapter 3 in Nonlinear Models in Mathematical Finance: Research Trends in Option Pricing NOVA SCIENCE PUBLISHERS, INC., ISBN 978-1160456-931-5, pp. 103-130,2009.

[Chmakova, 2005] Chmakova, A. Y. (2005). Symmetriereduktionen und explicite Lösungen für ein nichtlineares Modell eines Preisbildungsprozesses in illiquiden Märkten. PhD thesis, BTU Cottbus.

[Frey, 1998] Frey, R. (1998). Perfect option replication for a large trader. Finance Stochastic, 2:115-148. 


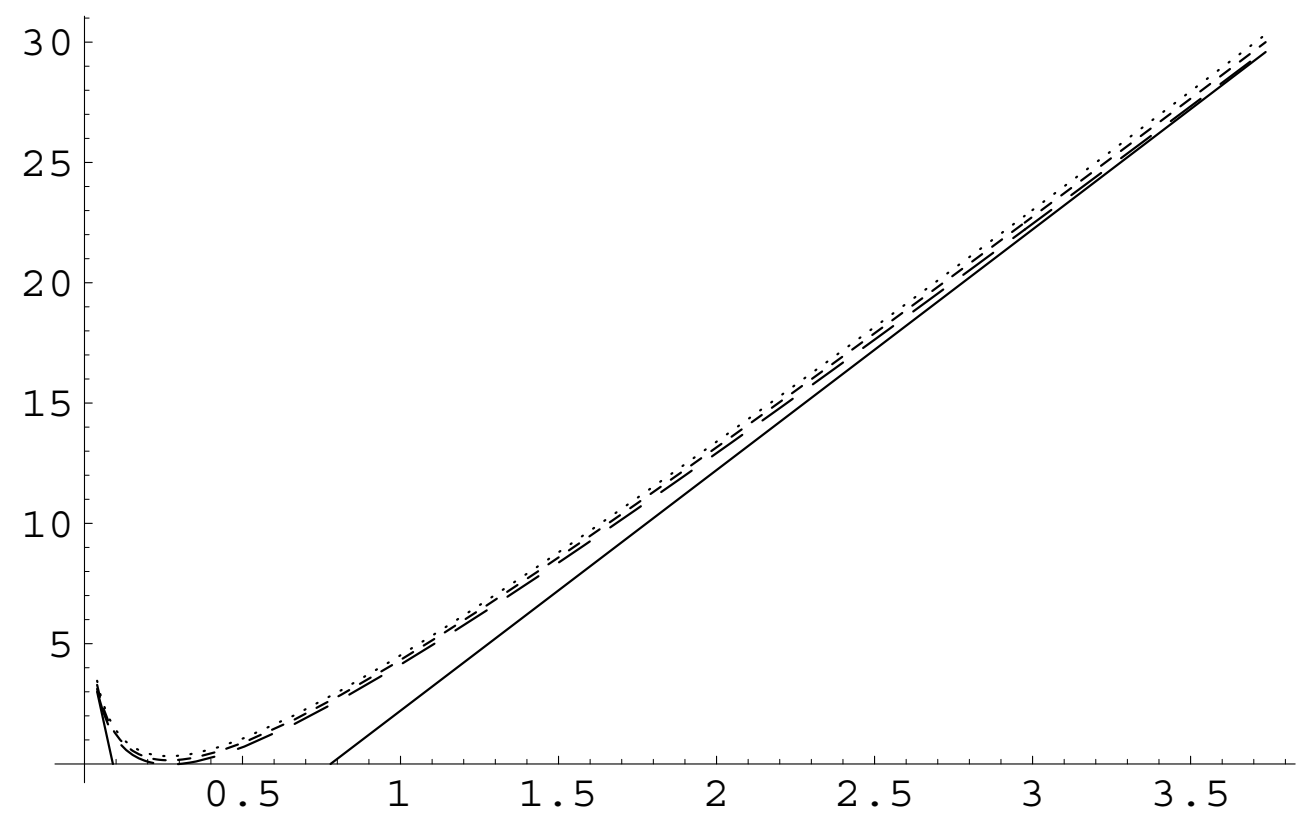

Figure 5: Plot of the solution $u_{r}(S, t)$ for the parameters $|c|=0.5, b=1.0, \sigma=0.3, d 1=$ $11.5, d 2=-9.0$. The variables $S, t$ lie in intervals $S \in(0.04,3.7)$ and $t=0$., (doted line), $t=5$., (short dashed line) and $t=10$., (long dashed line). Payoff for a long strip with 60 Puts and 10 Calls with exercise price 0.2 marked by thin solid line.

[Frey, 2000] Frey, R. (2000). Market illiquidity as a source of model risk in dynamic hedging. In R. Gibson, editor, Model Risk, Risk Publications, London, 2000, pages 125-136.

[Frey and Patie, 2002] Frey, R. and Patie, P. (2002). Risk management for derivatives in illiquid markets: a simulation study. In K. Sandmann and P. Schönbucher, editors, Advances in Finance and Stochastics, Springer, Berlin.

[Frey and Stremme, 1997] Frey, R. and Stremme, A. (1997). Market volatility and feedback effect from dynamic hedging. Mathematical Finance, 7(4):351-374.

[Gaeta, 1994] Gaeta, G. (1994). Nonlinear Symmetries and Nonlinear Equations, volume 299 of Mathematics and its Applications. Kluwer Academic Publishers, Dordrecht, NL Boston, USA London UK.

[Ibragimov, 1999] Ibragimov, N. H. (1999). Elementary Lie Group Analysis and Ordinary Differential Equations. John Wiley\&Sons, Chischester, USA New York, USA Weinheim, Germany Brisbane, USA Singapore, Singapore Toronto Canada etc.

[Lie, 1912] Lie, S. (1912). Vorlesungen über Differentialgleichungen mit bekannten infinitesimalen Transformationen. Teubner, Leipzig.

[Olver, 1986] Olver, P. J. (1986). Application of Lie groups to differential equations. Springer-Verlag, New York, USA.

[Ovsiannikov, 1982] Ovsiannikov, L. V. (1982). Group Analysis of Differential Equations. Academic Press, New York, USA.

[Schonbucher and Wilmott, 2000] Schonbucher, P. and Wilmott, P. (2000). The feedback effect of hedging in illiquid markets. SIAM J. Appl. Math., 61:232-272. 
[Shreve, 2004] Shreve, S. E. (2004). Stochastic Calculus for Finance II: Continuous-Time Models. Springer Finance. Springer-Verlag, New York, USA.

[Sircar and Papanicolaou, 1998] Sircar, K. and Papanicolaou, G. (1998). General Black-Scholes models accounting for increased market volatility from hedging strategies. Appl. Math. Finance, 5:45-82.

[Stephani, 1994] Stephani, H. (1994). Differential Gleichungen: Symmetrien und Lösungsmethoden. Spektrum Akademischer Verlag GmbH, Heidelberg, Germany. 